\title{
A two-stage amplified PZT sensor for monitoring lung and heart sounds in discharged pneumonia patients
}

Hongbin Chen', Shuai Yư ${ }^{2}$, Haiyang Liu ${ }^{3}$, Jie Liu ${ }^{3}$, Yongguang Xiao ${ }^{4}$, Dandan $\mathrm{Wu}^{1}$, Xiaoyu Pan ${ }^{5}$, Cuihong Zhou ${ }^{5}$, Yifeng Lei ${ }^{3}$ and Sheng Liu (B) 3,6

\begin{abstract}
Assessment of lung and heart states is of critical importance for patients with pneumonia. In this study, we present a small-sized and ultrasensitive accelerometer for continuous monitoring of lung and heart sounds to evaluate the lung and heart states of patients. Based on two-stage amplification, which consists of an asymmetric gapped cantilever and a charge amplifier, our accelerometer exhibited an extremely high ratio of sensitivity to noise compared with conventional structures. Our sensor achieves a high sensitivity of $9.2 \mathrm{~V} / \mathrm{g}$ at frequencies less than $1000 \mathrm{~Hz}$, making it suitable to use to monitor weak physiological signals, including heart and lung sounds. For the first time, lung injury, heart injury, and both lung and heart injuries in discharged pneumonia patients were revealed by our sensor device. Our sound sensor also successfully tracked the recovery course of the discharged pneumonia patients. Over time, the lung and heart states of the patients gradually improved after discharge. Our observations were in good agreement with clinical reports. Compared with conventional medical instruments, our sensor device provides rapid and highly sensitive detection of lung and heart sounds, which greatly helps in the evaluation of lung and heart states of pneumonia patients. This sensor provides a cost-effective alternative approach to the diagnosis and prognosis of pneumonia and has the potential for clinical and home-use health monitoring.
\end{abstract}

\section{Introduction}

Assessment of lung and heart states is critical when evaluating the health condition of patients with pneumonia. Lung injury in patients can be revealed by abnormal findings based on chest CT images ${ }^{1-3}$, PET/ $\mathrm{CT}^{4}$, and artificial intelligence (AI)-assisted diagnosis ${ }^{5,6}$. Lung ultrasound also offers a quantitative method to assess the lung state in patients ${ }^{7}$. Meanwhile, heart injury in patients can be revealed by echocardiography (ECG) ${ }^{7}$ and cardiac magnetic resonance imaging $(\mathrm{MRI})^{8,9}$.

\footnotetext{
Correspondence: Yifeng Lei (yifenglei@whu.edu.cn) or

Sheng Liu (shengliu@whu.edu.cn)

'Department of Pulmonary and Critical Care Medicine, Renmin Hospital of

Wuhan University, Wuhan 430060, China

${ }^{2}$ School of Mechanical Science and Engineering, Huazhong University of

Science and Technology, Wuhan 430074, China

Full list of author information is available at the end of the article

These authors contributed equally: Hongbin Chen, Shuai Yu
}

However, these methods generally require large, sophisticated, and expensive instruments; highly trained personnel; complex procedures; and far less harmless procedures (such as CT and MRI). Therefore, the development of novel sensing systems that are time-saving, low cost, highly sensitive, easy to read, instrument-free, and able to achieve on-site continuous monitoring ${ }^{10,11}$ has great potential in the diagnosis and prognosis of pneumonia diseases.

Auscultation of chest wall sounds, including both heart and lung sounds, offers an easy but very effective approach for the clinical diagnosis of cardiovascular and respiratory systems. Conventional stethoscopy is widely used for intermittent auscultation; however, stethoscopy has a number of limitations, such as poor wearability due to its bulky size, friction noise during diagnosis, and difficulty in detecting weak acoustic signals including lung

\section{(c) The Author(s) 2021}

(c) (i) Open Access This article is licensed under a Creative Commons Attribution 4.0 International License, which permits use, sharing, adaptation, distribution and reproduction cc) in any medium or format, as long as you give appropriate credit to the original author(s) and the source, provide a link to the Creative Commons license, and indicate if changes were made. The images or other third party material in this article are included in the article's Creative Commons license, unless indicated otherwise in a credit line to the material. If material is not included in the article's Creative Commons license and your intended use is not permitted by statutory regulation or exceeds the permitted use, you will need to obtain permission directly from the copyright holder. To view a copy of this license, visit http://creativecommons.org/licenses/by/4.0/. 
sounds. An alternative approach for detecting lung and heart sounds is based on accelerometer use ${ }^{12,13}$. Compared with stethoscopy, miniaturized accelerometers can be taped on a person's chest wall for more convenient and continuous cardiorespiratory monitoring. Previously, based on asymmetric gapped cantilever structures, we developed a series of small-sized and ultrasensitive sound sensors for continuous monitoring of heart and lung sounds in healthy subjects ${ }^{14-16}$. However, none of them have been systematically used to monitor patients with pneumonia.

Herein, we were motivated to explore more applications of our sound sensors in the assessment of lung and heart states of discharged pneumonia patients. From both theoretical simulations and mechanical tests, our sensors show improved sensitivity compared with conventional sensors, making them suitable for monitoring weak heart and lung sounds. Moreover, the lung and heart sound recorded by our sensors are in good agreement with previous clinical reports, suggesting that our sensor offers a potential alternative for the diagnosis and prognosis of pneumonia or other similar diseases.

\section{Results and discussion}

\section{Sensor structure and working principles}

In this study, we used a self-developed sound sensor with high sensitivity for continuous monitoring of lung and heart sounds (Fig. 1a, b). The sound sensor was based on a novel asymmetric gapped cantilever structure (Fig. 1c, d), which was composed with a piezoelectric beam made of piezoelectric ceramic materials of lead zirconium titanate (PZT) as the top layer, a bottom mechanical layer separated by a gap, and a movable proof mass made of aluminum (Table 1). The piezoelectric layer could convert biomechanical energy (such as acoustic vibration) to electric energy due to the piezoelectric effect $^{17,18}$. The mechanical beam could strengthen the stiffness of the whole cantilever (Fig. 1d). Furthermore, the output of the sound signal was amplified using an amplifier circuit (Fig. 1e).

\section{Theoretical simulation and characterization of sensor performance}

We used theoretical simulation to show the advantage of our sensor with an asymmetric gapped cantilever structure compared with conventional structures (Fig. S1). Harmonic response analysis of the dynamic model was conducted under different excitation accelerations (from $0.01 \mathrm{~g}$ to $0.11 \mathrm{~g}$ ). According to theoretical simulation, the strain experienced by the piezoelectric beam on our structure (Fig. 2a) was much more significant than that on a conventional structure (Fig. 2b). In our asymmetric gapped cantilever structure, the amplitude-frequency response showed that the maximum strain on the piezoelectric beam was $1.38 \times 10^{-4}$ under $0.11 \mathrm{~g}$ excitation (Fig. 2c). In contrast, the maximum strain on the piezoelectric beam of the conventional structure was only $1.42 \times 10^{-5}$ under the same excitation (Fig. 2d). Therefore, according to theoretical simulation, our structure produced a ten times higher strain on the piezoelectric beams than the conventional structure (Fig. 2).
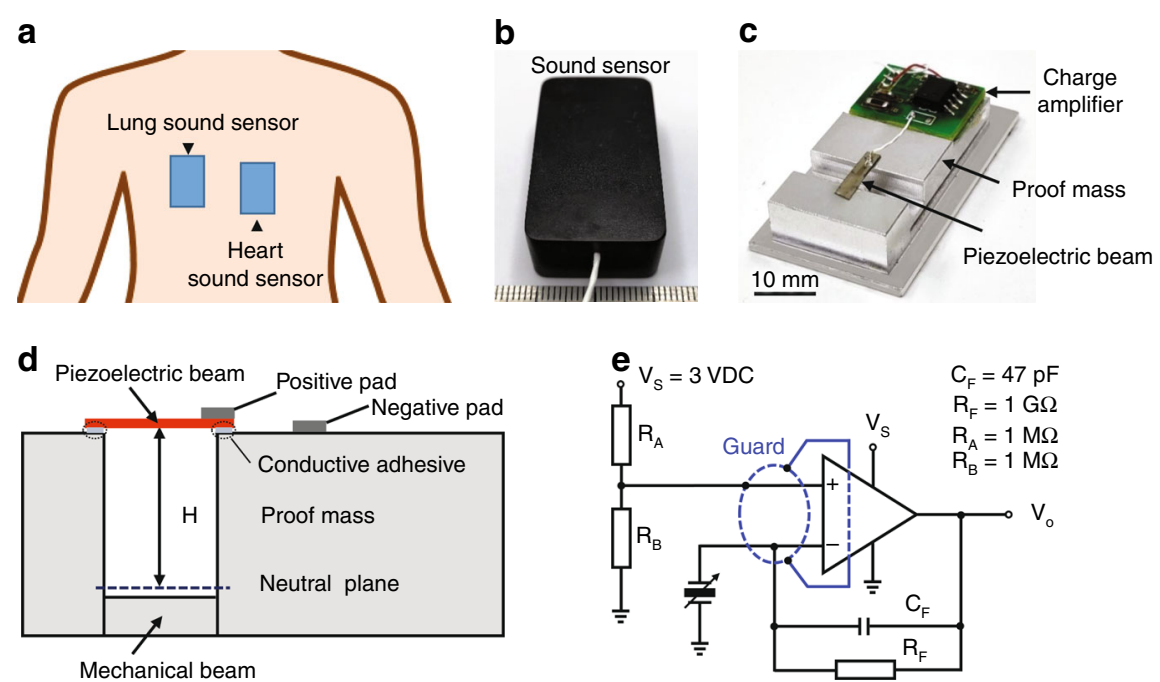

Fig. 1 Cardiorespiratory sound sensors and sensing mechanism. a Illustration of the placement of sensors on the human body for lung and heart sound monitoring. $\mathbf{b}$ Image of the prototype of the sound sensor. $\mathbf{c}$ Inside view of the prototype with a printed circuit board. $\mathbf{d}$ Structure of the accelerometer-based on an asymmetric gapped cantilever structure. $H$ is the distance between the layer of the piezoelectric beam and the neutral plane. e Built-in charge amplifier circuit for amplification of the piezoelectric signal 
Table 1 Parameters of materials used for our sensor

\begin{tabular}{lllll}
\hline & Materials & Density $\left(\mathbf{k g} / \mathbf{m}^{3}\right)$ & Young's modulus $(\mathrm{GPa})$ & Size $(\mathbf{m m})$ \\
\hline Piezoelectric beam & PZT & $7.8 \times 10^{3}$ & 66 & $3 \times 1 \times 0.127$ \\
Mechanical beam & Aluminum & $2.7 \times 10^{3}$ & 69 & $3 \times 12 \times 0.38$ \\
Proof mass & Aluminum & $2.7 \times 10^{3}$ & 69 & $20 \times 12 \times 1.5$ \\
\hline
\end{tabular}

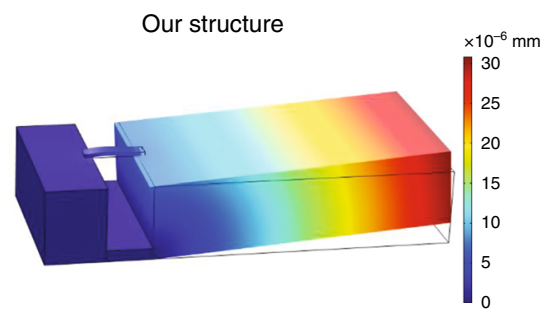

C

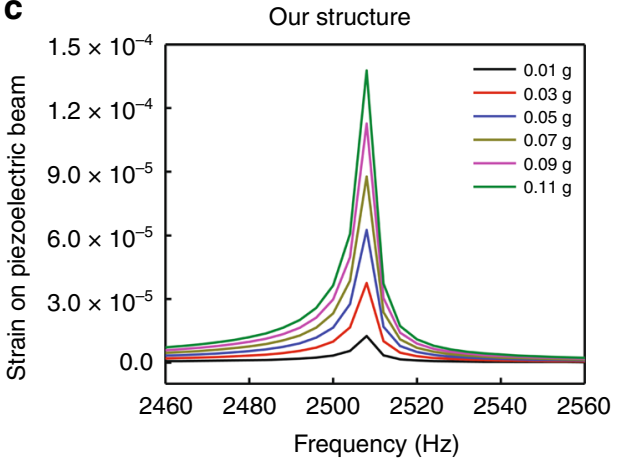

b

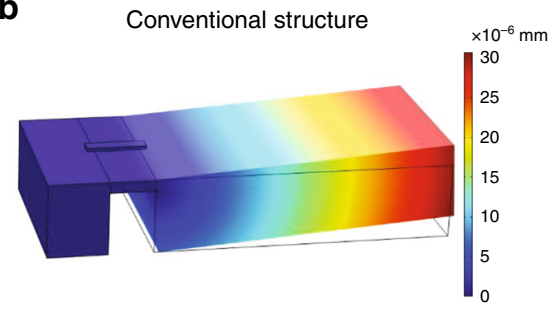

d

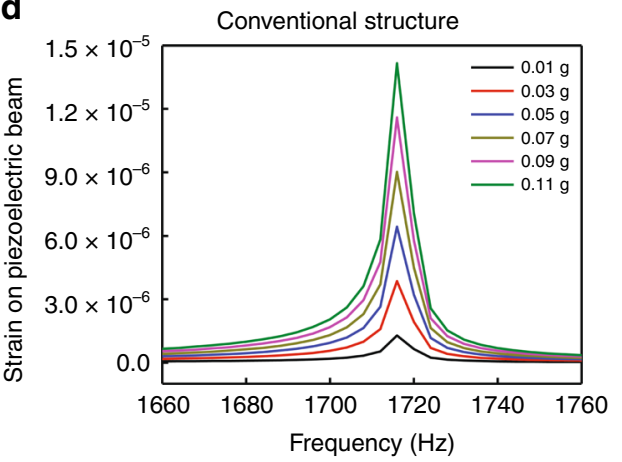

Fig. 2 Theoretical simulation of sensor performance with different structures. $\mathbf{a}$, $\mathbf{b}$ Total displacement of the sensor with our structure (a) or with a conventional structure (b); the excitation is $0.05 \mathrm{~g}$. c, d Amplitude-frequency response of the sensor with our structure (c) or with a conventional structure (d); different excitation forces are simulated

We then plotted the strain-excitation response of different structures (Fig. 3a). The sensitivity of the accelerometer can be defined by the equation below:

$$
\text { sensitivity }=\frac{\text { strain of piezoelectric beam }}{\text { excitation force }}
$$

Therefore, the sensitivity of the accelerometer can be calculated from the slope of the strain-excitation response (Fig. 3a), and the sensitivity of the accelerometer with our structure and conventional structure was calculated to be $1.25 \times 10^{-3}$ and $1.29 \times 10^{-4}(1 / \mathrm{g})$, respectively. The theoretical simulation indicates that the sensitivity of our sensor structure was improved 9.7 times that of the accelerometer with a conventional structure.

The strain experienced by the piezoelectric layer is proportional to the distance $(H)$ between the top piezoelectric beam and the neutral plane (Fig. S1) ${ }^{19}$. This distance is much larger on our structure than that on a conventional cantilever-based accelerometer (Fig. S1).
This explained the significantly larger strain or higher sensitivity of our sensor compared with that of conventional accelerometers.

Moreover, due to the piezoelectric effect ${ }^{17,18}$ of PZT materials, the strain on the piezoelectric beam can be transferred into an electric charge. As the charge produced by the piezoelectric beam was very weak and could not be directly collected, we used a charge amplifier to transfer the charge into voltage and further amplify the signal (Fig. 1c, e); therefore, the sensitivity of the accelerometer can be expressed as $\mathrm{V} / \mathrm{g}$.

To avoid using balanced dual supplies to create op-amp circuits, the op-amp LMP7721 (Texas Instrument), which enables a single supply, was selected for the charge amplifier design (Fig. 1e). LMP7721 has an ultralow typical input bias current of $3 \mathrm{fA}$ and low voltage noise of $6.5 \mathrm{nV} / \sqrt{\mathrm{Hz}}$, making it ideal for amplifying high impedance signals. The average level of op-amp input was biased to $V_{S} / 2$ by the $R_{A}-R_{B}$ divider pair (Fig. 1e). The amplification rate of this circuit is inversely proportional 

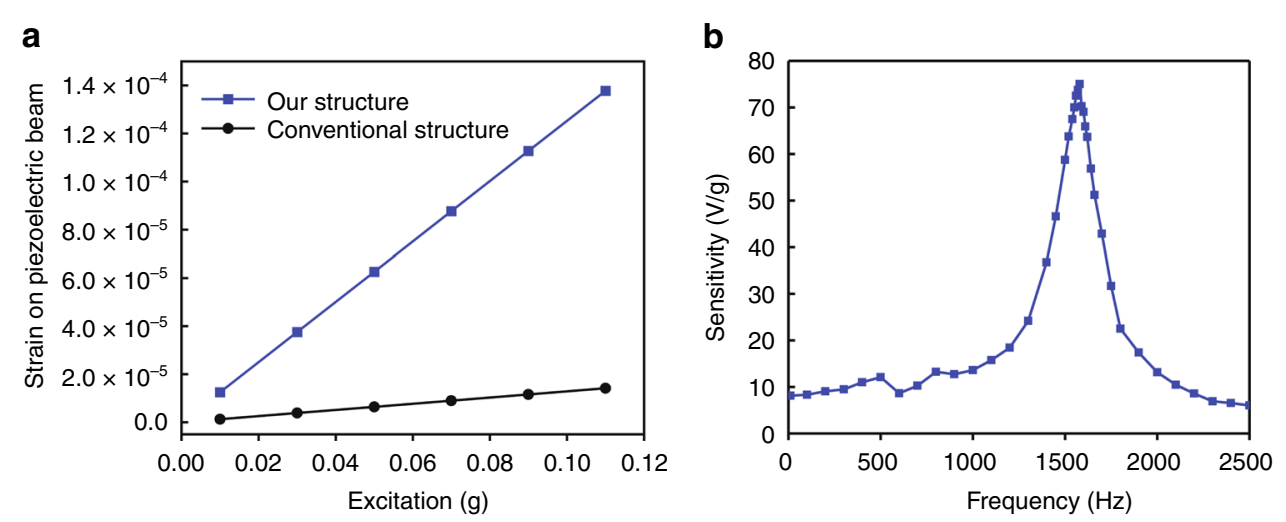

Fig. 3 Sensitivity of the sensors. a Sensitivity of sensors with different structures by theoretical simulation. $\mathbf{b}$ Sensitivity of our sensor measured on a mechanical shaker

to the feedback capacitance $\left(C_{F}\right)$. The signal-to-noise ratio (SNR) and lower cutoff frequency are also inversely proportional to the $C_{F}$. The $C_{F}$ in this charge amplifier was set as $47 \mathrm{pF}$ (Fig. 1e) at the tradeoff of charge amplification rate, SNR, and lower cutoff frequency. Since the input capacitance of the piezoelectric transducer is $\sim 1 \mathrm{nF}$, the amplification rate of the circuit was calculated to be 21.3. In addition, the charge amplifier circuit was designed with a $1 \mathrm{G} \Omega$ feedback resistor $\left(R_{F}\right)$ (Fig. 1e). Together, this circuit yielded a low cutoff frequency of $3.4 \mathrm{~Hz}$, making it satisfactory for heart and lung sound monitoring.

In addition, ultralow input bias current op-amp circuits require precautions to achieve the best performance. The leakage current on the surface of the circuit board could exceed the input bias current of the amplifier and could even be 100 times higher. To minimize surface leakage, a guard trace was designed to completely surround the input terminals and other circuitry connecting to the inputs of the op-amp (Fig. 1e).

\section{Measurement of the sensitivity and noise of our sensor}

We measured the sensitivity of our sensor on a mechanical shaker by setting a commercial accelerometer (752A13, Endevco) as the gold standard. The sensitivity-frequency response of our sensors is shown in Fig. 3b. Our sound sensor had a resonance frequency of $1600 \mathrm{~Hz}$ (Fig. 3b), which is higher than the frequency range of heart sounds $(20-400 \mathrm{~Hz})$ and lung sounds $(60-1000 \mathrm{~Hz})$.

As shown in Fig. 4a, within the sound frequency range from 20 to $1000 \mathrm{~Hz}$, the output voltage of our sensor increased when the excitation acceleration increased. The sensitivity of our sensor at $0.01 \mathrm{~g}, 0.05 \mathrm{~g}$, and $0.1 \mathrm{~g}$ under different excitation accelerations was calculated to be $9.1966 \mathrm{~V} / \mathrm{g}, 9.1982 \mathrm{~V} / \mathrm{g}$, and $9.252 \mathrm{~V} / \mathrm{g}$, respectively (Fig. 4a). These results proved that the sensitivity of our sensor was consistent $(\sim 9.2 \mathrm{~V} / \mathrm{g})$ under different excitation accelerations within the heart and lung sound frequency ranges.
By using the novel asymmetric gapped cantilever structure, the sensitivity of our sound sensor could achieve $9.2 \mathrm{~V} / \mathrm{g}$ at frequencies less than $1000 \mathrm{~Hz}$ (Figs. 3b and $4 \mathrm{a}$ ). The sensitivity of conventional piezoelectric accelerometers or MEMS-based accelerometers is generally less than $1 \mathrm{~V} / \mathrm{g}$ (Table 2$)^{20-22}$. Comparably, our sensor showed significantly improved sensitivity $(9.2 \mathrm{~V} / \mathrm{g})$ in frequencies less than $1000 \mathrm{~Hz}$. The enhanced sensitivity of our sensor makes it suitable for the detection of weak physiological sounds, such as lung and heart sounds, especially for weak lung sound detection ${ }^{23}$.

In addition, since sensor noise is another important characteristic, we measured the intrinsic noise of our sensor upon a vibration isolation mechanical shaker at midnight. As shown in Fig. 4b, the noise spectrum and the output voltage density demonstrated that the noise level of our sensor was $\sim 1 \mu \mathrm{V} / \sqrt{\mathrm{Hz}}$ within the frequency range of heart and lung sounds (from 20 to $1000 \mathrm{~Hz}$ ). Therefore, the lower noise limit of our sensor was calculated to be $109 \mathrm{ng} / \sqrt{\mathrm{Hz}}$.

From the lung sound signal spectrum (Fig. 4c), the output voltage density of the lung sound signal from 60 to $1000 \mathrm{~Hz}$ was $\sim 125 \mu \mathrm{V} / \sqrt{ } \mathrm{Hz}$ (Fig. 4c), which was 125 times higher than the intrinsic noise level. Similarly, from the heart sound signal spectrum (Fig. 4d), the output voltage density of the heart sound signal was $\sim 890 \mu \mathrm{V} / \sqrt{\mathrm{Hz}}$ from 20 to $400 \mathrm{~Hz}$ (Fig. 4d), which was 890 times higher than the intrinsic noise level.

The SNR can be calculated according to the equation below,

$$
\mathrm{SNR}=20 \log _{10}(V s / V n)
$$

where $V s$ and $V n$ represent the signal voltage and noise voltage, respectively. Therefore, the SNRs of the lung sound signal and heart sound signal was $42 \mathrm{~dB}$ and $59 \mathrm{~dB}$, respectively. The SNRs of our sensors are two times higher than those of commercial stethoscopes. 

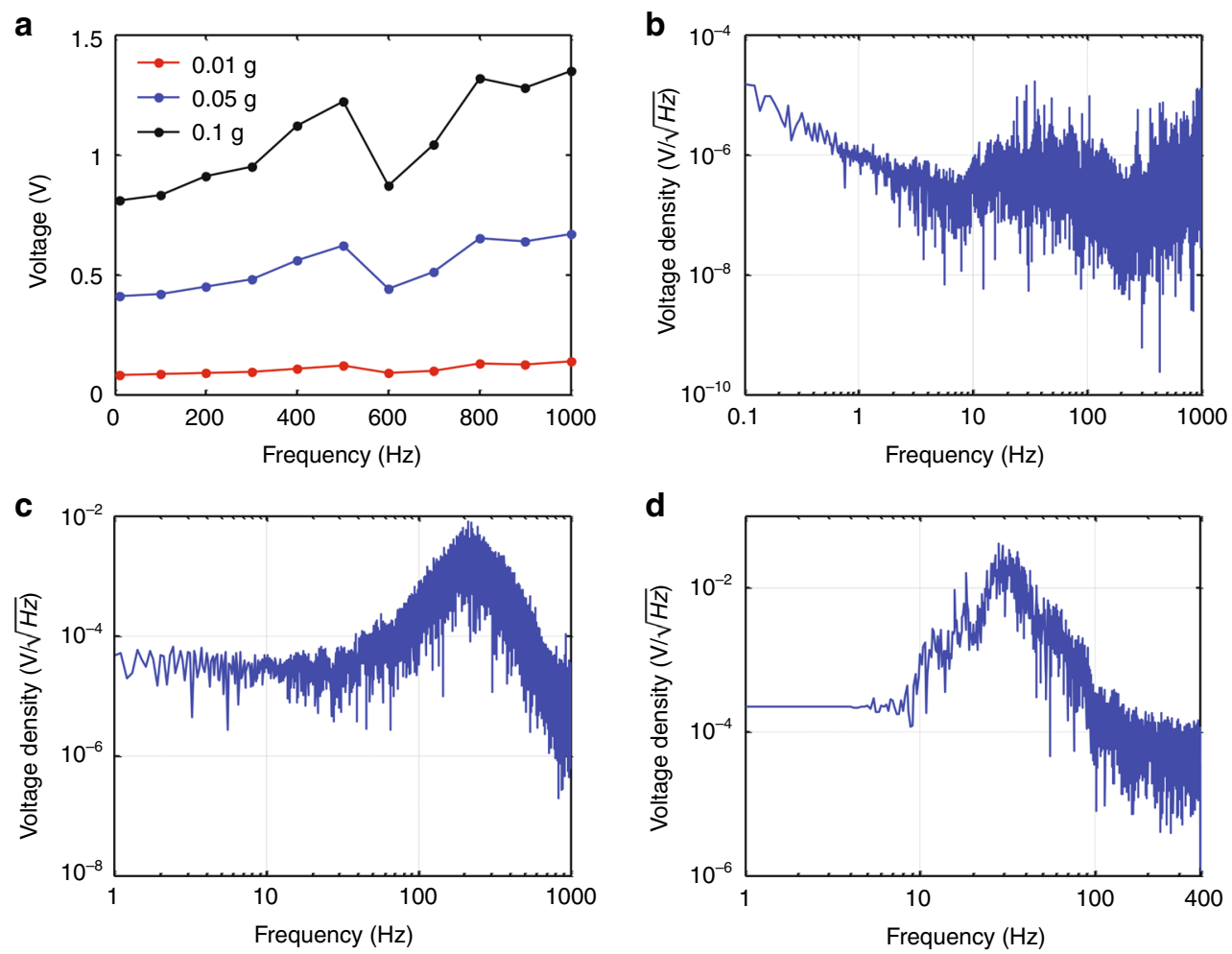

Fig. 4 Sensor performance at sound frequencies ranging from 20 to $1000 \mathrm{~Hz}$. a Output voltage response to different excitation accelerations. $\mathbf{b}$ Noise spectrum measured upon a vibration isolation mechanical shaker. $\mathbf{c}$ Lung sound signal spectrum and (d) heart sound signal spectrum measured on a healthy subject

Table 2 Comparison of different types of sound sensors

\begin{tabular}{|c|c|c|c|c|}
\hline Sensor & Mechanism & Size & Weight & Sensitivity \\
\hline Our sensor & Piezoelectric accelerometer & $\begin{array}{l}\text { l: } 39 \mathrm{~mm} \\
w: 23 \mathrm{~mm} \\
h: 13.5 \mathrm{~mm}\end{array}$ & $13.5 \mathrm{~g}$ & $9200 \mathrm{mV} / \mathrm{g}$ \\
\hline Endevco 752A12 & Piezoelectric accelerometer & $h: 23.6 \mathrm{~mm}$ & $13 \mathrm{~g}$ & $100 \mathrm{mV} / \mathrm{g}$ \\
\hline PCB 393 C & Piezoelectric accelerometer & $\begin{array}{l}\text { Ф: } 57.2 \mathrm{~mm} \\
h: 54.9 \mathrm{~mm}\end{array}$ & $885 \mathrm{~g}$ & $1000 \mathrm{mV} / \mathrm{g}$ \\
\hline Analog devices ADXL354 & MEMS accelerometer & $\begin{array}{l}\text { l: } 6 \mathrm{~mm} \\
w: 6 \mathrm{~mm} \\
h: 2.1 \mathrm{~mm}\end{array}$ & $0.26 \mathrm{~g}$ & $400 \mathrm{mv} / \mathrm{g}$ \\
\hline Bosch Sensortec BMA456 & MEMS accelerometer & $\begin{array}{l}\text { l: } 2 \mathrm{~mm} \\
w: 2 \mathrm{~mm} \\
h: 0.65 \mathrm{~mm}\end{array}$ & $<0.1 \mathrm{~g}$ & $300 \mathrm{mV} / \mathrm{g}$ \\
\hline
\end{tabular}

Sensor performance for lung and heart sound monitoring

Subsequently, we used our sound sensor device to monitor the lung and heart sounds of healthy volunteers in a regular laboratory environment to prove the auscultation ability of our sensor device.
Compared with a commercial high-end electric stethoscope based on a conventional cantilever structure, our sensor exhibited much better performance for recording both lung and heart signals, and especially for recording weak lung sounds (Fig. 5). Generally, lung sounds are 

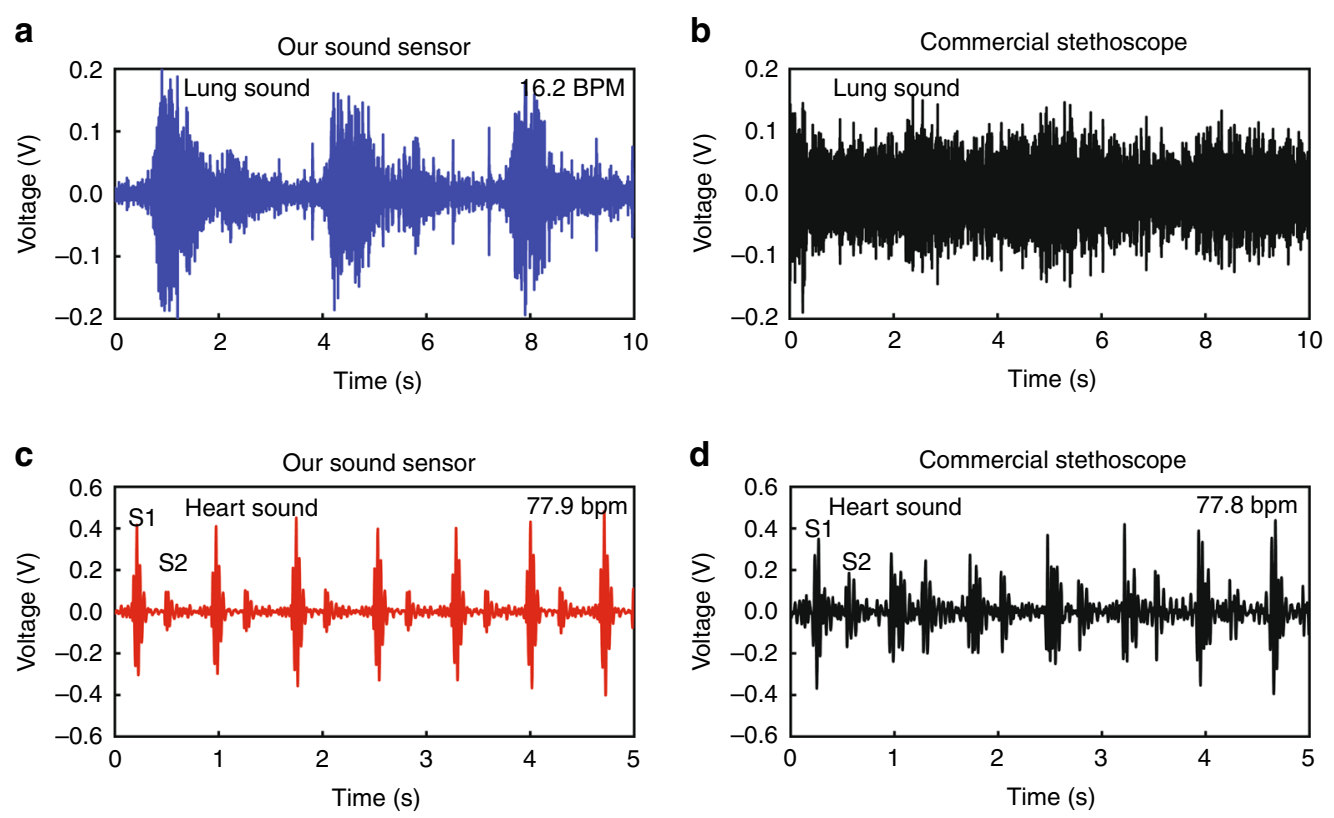

Fig. $\mathbf{5}$ Waveforms of lung and heart sounds monitored on a healthy volunteer. $\mathbf{a}$, $\mathbf{b}$ Lung sound waveform detected by our sound sensor (a) and by a commercial high-end electric stethoscope (b). c, $\mathbf{d}$ Heart sound signal recorded by our sensor (c) and by commercial stethoscopy (d). The volunteer was a 29-year-old healthy male (\#1), and sound signals were recorded during a resting respiratory function

much weaker than heart sounds during regular breathing $^{23}$; therefore, lung sounds, especially for gentle breathing, are difficult to detect. However, with the asymmetric gapped cantilever structure, our sensor can indeed detect weak lung sounds with a high SNR (Fig. 5a, Audio S1). In contrast, the commercialized high-end electronic stethoscope, which is based on a conventional cantilever structure, can hardly distinguish lung sounds from the noise of the captured signal (Fig. 5b). The respiratory rate of the measured volunteer was 16.2 breaths per minute (hereafter "BPM") (Fig. 5a, Table 3), which was in the normal range of resting respiratory rates (12-20 BPM). Moreover, the lung sounds measured from different healthy volunteers were consistently within the normal respiratory rate range (Table 3 ).

For heart sound monitoring, the SNR of the heart sounds detected by our sensor was two times higher than that of a commercial stethoscope (Fig. 5c, d). We could clearly distinguish two normal heart sounds from the obtained heart sound waveform, including the first heart sound (S1) and the second heart sound (S2) (Fig. 5c, Audio S2), which correspond to the "lub" and "dub" sounds of a heartbeat, produced by the closure of the atrioventricular valves and semilunar valves, respectively $^{24}$. The heart rate of the measured healthy volunteer was 77.9 beats per minute (hereafter "bpm") (Fig. $5 \mathrm{c}$ and Table 3), which was in the normal heart rate range of adults $(60-88 \mathrm{bpm})$. Moreover, the heart sounds from different healthy volunteers were consistently within the normal range of heart rates (Table 3).

The measurements of healthy volunteers proved that our sound sensor could effectively detect lung and heart sounds in the human body (Table 3), especially relatively weak lung sounds (Fig. 5a). It is also worth noting that these measurements were carried out in a regular laboratory environment full of airborne noise. These results proved that our sensor was not very sensitive to airborne noise and can therefore be applied in medical applications.

\section{Sensor monitoring of patients with pneumonia Classification of lung and heart sounds of patients}

We monitored the lung and heart sounds of discharged pneumonia patients during their follow-up visit to the hospital to evaluate their lung and heart states.

According to the sensor monitoring and based on the clinical diagnosis of the discharged pneumonia patients, we found four typical characteristics from the recorded sound signals (Table 4) independent of the patients' sex, age, preexisting conditions, the severity of illness, and time from the original diagnosis. The four types were typing I, patients with normal respiratory rate and normal heart rate; type II, patients with shortness of respiratory but normal heart rate; type III, patients with the normal respiratory rate but high heart rate; and type IV, patients with shortness of respiratory and high heart rate (Table 4). 
Table 3 Monitoring of lung and heart sounds in healthy volunteers by our sound sensor

\begin{tabular}{lll}
\hline Healthy volunteers & $\begin{array}{l}\text { Respiratory rate by } \\
\text { sensor (BPM) }\end{array}$ & $\begin{array}{l}\text { Heart rate by } \\
\text { sensor (bpm) }\end{array}$ \\
\hline$\# 1$ & 16.2 & 77.9 \\
$\# 2$ & 17.1 & 72.3 \\
$\# 3$ & 15.8 & 69.0 \\
$\# 4$ & 18.2 & 75.0 \\
$\# 5$ & 15.4 & 70.6 \\
\hline
\end{tabular}

Table 4 Definition of four types of lung and heart states in patients

\begin{tabular}{lll}
\hline & Respiratory rate (BPM) & Heart rate $(\mathbf{b p m})$ \\
\hline Type I & $12-20$ & $60-88$ \\
Type II & $>20$ & $60-88$ \\
Type III & $12-20$ & $>88$ \\
Type IV & $>20$ & $>88$ \\
\hline
\end{tabular}

Generally, a decreased respiratory rate is a good sign for healthy adults ${ }^{23}$, and the heart rates of some athletes or those who often exercise may be lower than those of ordinary adults ${ }^{23}$, which may explain why a decreased respiratory rate or decreased heart rate were hardly found in discharged pneumonia patients in this study.

\section{Characterization of lung and heart sounds of patients}

We described our findings of these four types of lung and heart sounds in detail.

Type I patients exhibited both a normal respiratory rate and normal heart rate (Fig. 6a, Audio S3-S4). From our sensor monitoring, both the respiratory rate and heart rate of the patient were in normal ranges (Fig. $6 \mathrm{a}_{\mathrm{i}}, \mathrm{a}_{\mathrm{ii}}$, Table S1). We found that the lung and heart sounds of most discharged patients (29/41) exhibited type I characteristics, with an average respiratory rate of $16.5 \pm 1.8 \mathrm{BPM}$ and an average heart rate of $70.9 \pm 6.5 \mathrm{bpm}$ (Table 5), where the latter showed no significant difference from the ECG data $(p>$ 0.05 , Table 5). This result indicated that most discharged patients $(70.7 \%)$ recovered from pneumonia and exhibited good lung and heart functions after discharge (Table 5).

Type II patients showed shortness of respiratory function but normal heart rates (Fig. 6b, Audio S5-S6). From our sensor monitoring, the respiratory rate of the monitored patient increased to $24.0 \mathrm{BPM}\left(\mathrm{Fig} .6 \mathrm{~b}_{\mathrm{i}}\right.$ ), and the heart rate of the patient was $68.2 \mathrm{bpm}$ (Fig. $6 \mathrm{~b}_{\mathrm{ii}}$, Table S1). We found 6/ 41 discharged patients with type II characteristics (Table 5), with an average respiratory rate of $22.9 \pm 1.5 \mathrm{BPM}$ and an averaged heart rate of $75.9 \pm 6.4 \mathrm{bpm}$ (Table 5), respectively.
The measured heart rates were in good accordance with the ECG data $(p>0.05$, Table 5$)$. This result showed that $14.6 \%$ of the discharged patients exhibited normal heart function but still suffered from impaired lung function due to pneumonia infection (Table 5).

Type III patients exhibited a normal respiratory rate but an increased heart rate (Fig. 6c, Audio S7-S8). Our sensor monitoring showed that these patients exhibited a normal respiratory rate (13.3 BPM) (Fig. $6 \mathrm{c}_{\mathrm{i}}$ ) but with an increased heart rate $(89.6 \mathrm{bpm})$ (Fig. $6 \mathrm{c}_{\mathrm{i} i}$, Table S1). From our sensor monitoring, we found $2 / 41$ patients with type III characteristics (Table 5), with an average respiratory rate and heart rate of $12.9 \pm 0.6 \mathrm{BPM}$ and $88.9 \pm 0.9 \mathrm{bpm}$, respectively (Table 5). These observations indicate that $4.9 \%$ of the discharged patients exhibited recovered lung function but still faced a critical challenge of heart injury (Table 5).

Type IV patients showed the worst recovery and exhibited both shortness of respiratory function and increased heart rate (Fig. 6d, Audio S9-S10). Our sensor monitoring showed that a patient's respiratory rate increased to $30.0 \mathrm{BPM}$ (Fig. $6 \mathrm{~d}_{\mathrm{i}}$ ) and the patient's heart rate increased to as high as $113.2 \mathrm{bpm}$ (Fig. $6 \mathrm{~d}_{\mathrm{ii}}$ and Table S1). We found 4/41 patients with type IV characteristics (Table 5), with an average respiratory rate of $30.0 \pm 1.2 \mathrm{BPM}$, and an averaged heart rate of $98.8 \pm 10.7 \mathrm{bpm}$ (Table 5). These results indicated that $9.8 \%$ of discharged patients had very poor recovery and suffered from both heart injury and lung injury after pneumonia infection (Table 5).

Generally, lung injury during pneumonia infection is revealed by chest $\mathrm{CT}$ imaging ${ }^{2,3}$ and lung ultrasound ${ }^{7}$. Even after patients with pneumonia are discharged, patients may suffer from a lung injuries, such as lung fibrosis and changes in lung function ${ }^{1}$. Compared with sophisticated CT or lung ultrasound instruments, our small-sized sound sensor provided a fast and effective evaluation of lung function of the patients and revealed that $24.4 \%$ of the discharged pneumonia patients had lung injury in terms of shortness of respiratory function (type II and IV, Table 5).

In addition, pneumonia prominently affects the cardiovascular system of patients ${ }^{8}$. The presence of cardiac injury and myocardial inflammation in patients recovered from pneumonia was revealed by $\mathrm{ECG}^{7}$ and cardiovascular MRI ${ }^{9}$. From the heart sounds measured by our sound sensor, we also revealed heart injury in $14.7 \%$ of the discharged patients (type III and IV, Table 5). Compared with conventional ECG and cardiac MRI, our sensor provides a simple, easy but very effective approach to evaluate heart injury in discharged patients.

\section{Time course tracking of the lung and heart state of a pneumonia patient}

We next tracked the lung and heart sounds of a patient at different time points after discharge to evaluate the time evolution of the lung and heart states of the patient. 

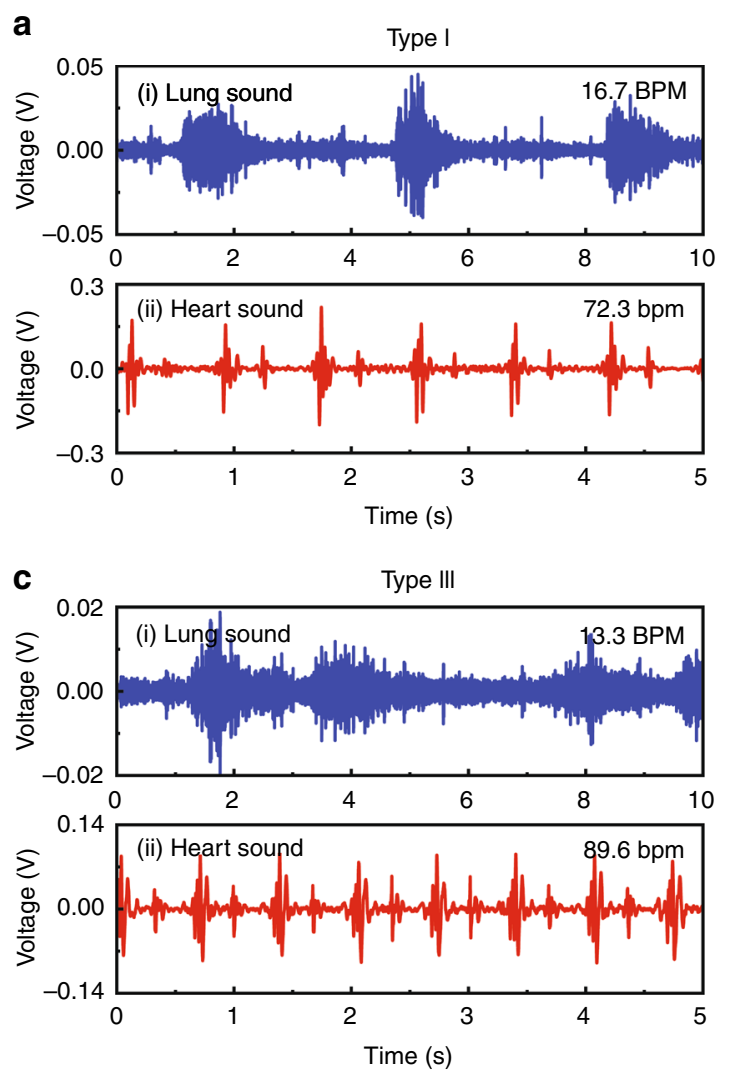
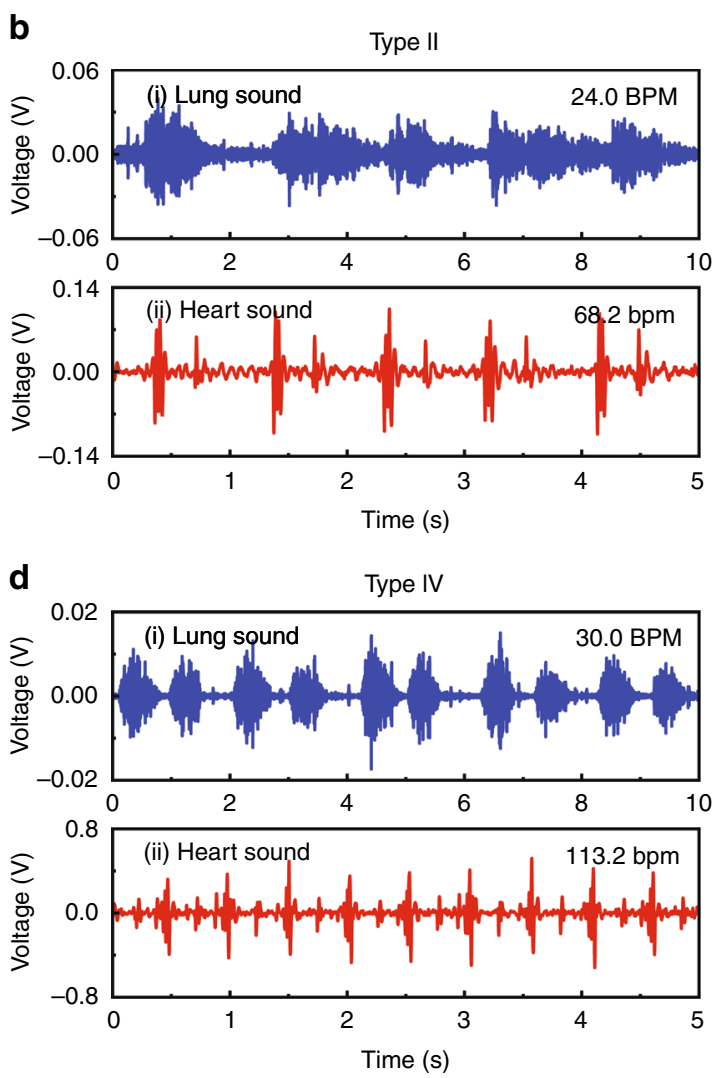

Fig. 6 Four types of lung and heart sound recorded from discharged pneumonia patients. a Records of a patient with a normal respiratory rate and normal heart rate (\#31). $\mathbf{b}$ Records of a patient with shortness of breath but a normal heart rate (\#3). $\mathbf{c}$ Records of a patient with a normal respiratory rate but a high heart rate (\#16). $\mathbf{d}$ Records of a patient with shortness of breath and a high heart rate (\#4). $\mathbf{a}_{\mathbf{i}}, \mathbf{b}_{\mathbf{i}}, \mathbf{c}_{\mathbf{i}}, \mathbf{d}_{\mathbf{i}}$ Lung sounds detected by our sensor; $\mathbf{a}_{\mathbf{i i}}, \mathbf{b}_{\mathbf{i}}, \mathbf{c}_{\mathbf{i i}}, \mathbf{d}_{\mathbf{i i}}$ Heart sounds recorded by our sensor

Over time, from our sound sensor monitoring, we found that the lung and heart function of the monitored patient gradually improved (Fig. 7). From the lung sound waveforms, the respiratory rate of the monitored patient decreased from 23.1 to 18.8 and then to $16.2 \mathrm{BPM}$ on different dates (Fig. $7 \mathrm{a}_{\mathrm{i}}, \mathrm{b}_{\mathrm{i}}, \mathrm{c}_{\mathrm{i}}, \mathrm{d}$ ), changing from shortness of breath to a normal respiratory rate. These observations suggested that the lung function of the patient gradually improved to a normal state. Moreover, from the monitoring of heart sounds, the heart rate of the patient was 83.3, 76.9, and $74.1 \mathrm{bpm}$ on different dates, respectively (Fig. $7 \mathrm{a}_{\mathrm{ii}}, \mathrm{b}_{\mathrm{ii}}, \quad \mathrm{c}_{\mathrm{ii}}$, e and Table S2), indicating the improvement of the heart states of the patient.

\section{Time evaluation of lung and heart states of 41 pneumonia patients}

We then investigated the time evolution of lung and heart states of discharged pneumonia patients $(n=41)$. During the first monitoring session on 15 June 2020, the ratio of four types of patients was evenly distributed (Tables S3-S4 and Fig. S2a). Over time, the ratio of type I patients increased, whereas the ratio of type II, III, and IV patients decreased (Fig. S2a). As time went by, the accumulated ratio of type I patients gradually increased from $25.0 \%$ to $70.7 \%$, whereas the accumulated ratios of type II, III, and IV patients gradually decreased (Tables S5-S6 and Fig. S2b).

From our sound sensor monitoring, we proved that the lung and heart injuries in pneumonia patients gradually decreased after discharge, and the lung and heart functions of the patients gradually improved over time. The results of our sensor monitoring were in agreement with clinical observations that pneumonia patients can suffer long-term lung and heart damage, but their condition tends to improve over time ${ }^{25}$.

Based on the above results from sound sensor monitoring, we found four typical characteristics in discharged pneumonia patients (Tables 4 and 5 and Fig. 6), and we found lung injury (14.6\%), heart injury (4.9\%), and both lung and heart injury (9.8\%) is discharged patients (Table 5). Our results were consistent with ECG data (Table 5) and clinical observations based on chest $\mathrm{CT}^{2,3}$ and cardiac MRI ${ }^{8,9}$. With our sensor device, we successfully tracked the recovery course of the pneumonia 
Table 5 Four types of lung and heart states recorded in discharged pneumonia patients

\begin{tabular}{lllllll}
\hline & $\begin{array}{l}\text { Respiratory rate by } \\
\text { sensor (BPM) }\end{array}$ & $\begin{array}{l}\text { Heart rate by } \\
\text { sensor }(\mathbf{b p m})\end{array}$ & $\begin{array}{l}\text { Number of } \\
\text { patients }\end{array}$ & $\begin{array}{l}\text { Ratio of } \\
\text { patients }\end{array}$ & $\begin{array}{l}\text { Heart rate by } \\
\text { ECG (bpm) }\end{array}$ & $\begin{array}{l}\boldsymbol{p} \text { value among } \\
\text { heart rates }\end{array}$ \\
\hline Type I & $16.5 \pm 1.8$ & $70.9 \pm 6.5$ & 29 & $70.7 \%$ & $71.7 \pm 6.4$ & 0.0718 \\
Type II & $22.9 \pm 1.5$ & $75.9 \pm 6.4$ & 6 & $14.6 \%$ & $76.2 \pm 5.6$ & 0.0504 \\
Type III & $12.9 \pm 0.6$ & $88.9 \pm 0.9$ & 2 & $4.9 \%$ & $89.5 \pm 3.5$ & 0.0525 \\
Type IV & $30.0 \pm 1.2$ & $98.8 \pm 10.7$ & 4 & $9.8 \%$ & $97.8 \pm 8.1$ & 0.0505 \\
\hline
\end{tabular}
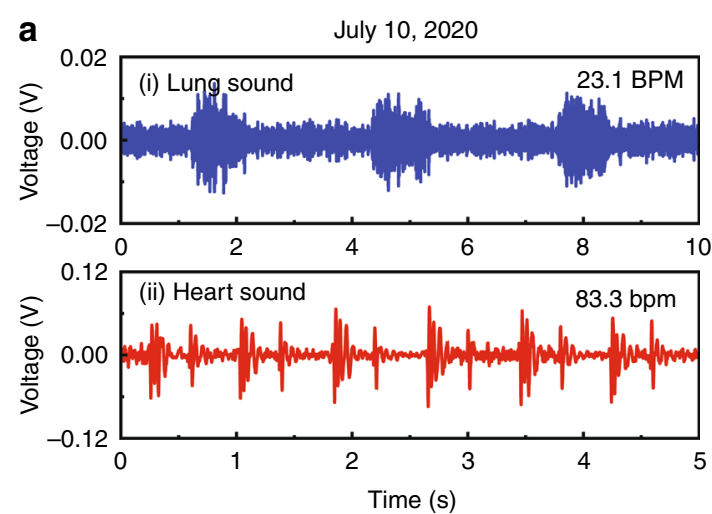

C
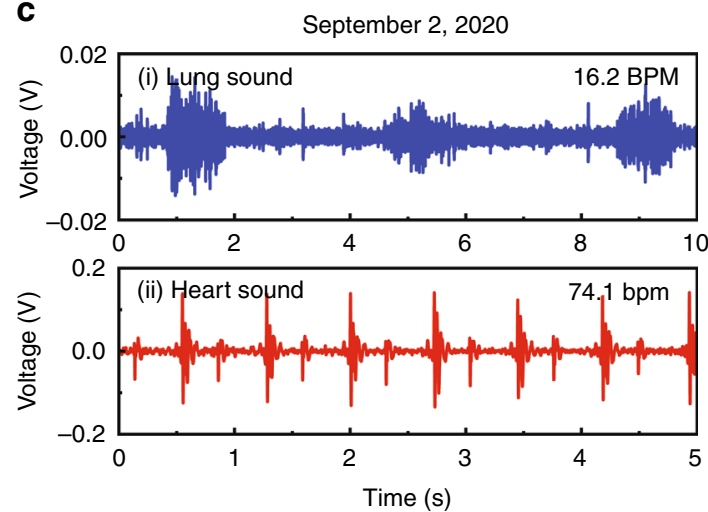
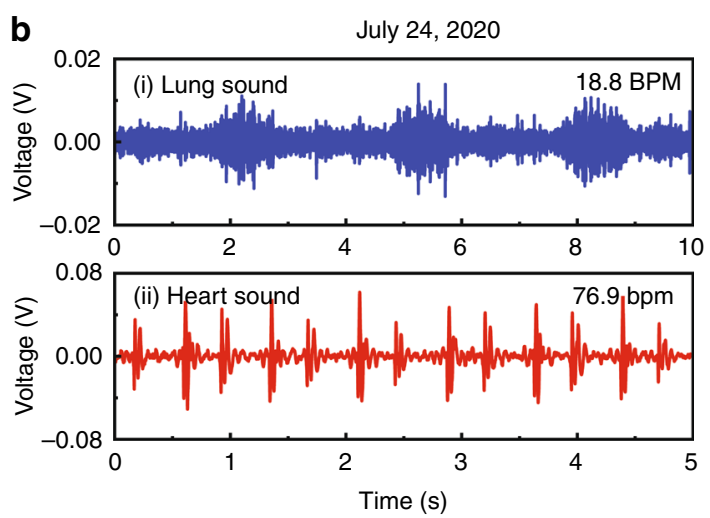

d
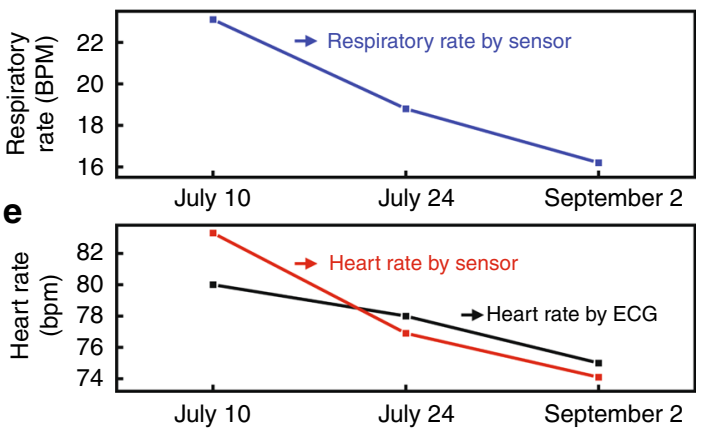

Fig. 7 Time evolution of lung and heart states of a discharged pneumonia patient. $\mathbf{a}, \mathbf{b}$, $\mathbf{c}$ Sound sensor monitoring of the patient (\#23) on July 10 (a), July $24(\mathbf{b})$, and September 02, $2020(\mathbf{c})$. $\mathbf{a}_{\mathbf{i}}, \mathbf{b}_{\mathbf{i}}, \mathbf{c}_{\mathbf{i}}$ Lung sounds recorded by our sensor; $\mathbf{a}_{\mathbf{i i}}, \mathbf{b}_{\mathbf{i i}}, \mathbf{c}_{\mathbf{i i}}$ Heart sounds detected by our sensor. $\mathbf{d}$ Time evolution of lung sounds of the patient detected by sensor monitoring. e Time evolution of heart sounds of the patient detected by our sound sensor monitoring (red curve) compared with ECG data (black curve)

patients. Over time, the lung and heart states of the patients gradually improved after discharge (Figs. 7 and S2), and our sound sensor observations were in good agreement with the clinically reported tendency ${ }^{25}$.

Compared with conventional large, sophisticated and expensive instruments, our small-sized sensor provides a rapid, simple, highly sensitive approach to detect lung and heart sounds, which greatly helps the evaluation of lung and heart states of pneumonia patients and provides an alternative approach for the diagnosis and prognosis of pneumonia disease. Moreover, our sensor provides a robust approach to capture lung and heart sounds, where patients can reliably obtain the same high-quality signals as trained medical personnel; therefore, our sensor has great potential for clinical use as well as home-use health monitoring, especially in the field of wearable electronics $^{26,27}$.

\section{Conclusions}

In this study, we developed a two-stage amplified PZT sensor for lung and heart sound monitoring in discharged pneumonia patients. Benefiting from the asymmetric 
gapped cantilever structure and built-in charge amplifier circuit, our accelerometer exhibited an extremely high ratio of sensitivity to noise compared to commercialized accelerometers. In addition, a sensitivity of $9.2 \mathrm{~V} / \mathrm{g}$ at a frequency less than $1000 \mathrm{~Hz}$ was achieved by our sensor, making it suitable for weak lung and heart sound monitoring. We used our ultrasensitive sound sensor to study the lung and heart states of discharged pneumonia patients. According to our sensor monitoring, for the first time, we classified the discharged pneumonia patients into four types: patients with a normal respiratory rate and normal heart rate, patients with shortness of breath but a normal heart rate, patients with a normal respiratory rate but high heart rate, and patients with shortness of breath and a high heart rate, which represented $70.7 \%, 14.6 \%, 4.9 \%$ and $9.8 \%$ of the discharged patients, respectively. With our sound sensor, we successfully tracked the recovery course of pneumonia patients. Over time, the lung and heart function of the patients gradually improved to normal performance after discharge. Compared with conventional medical instruments, our small-sized sensor provides a rapid, simple, and highly sensitive detection of lung and heart sounds, which greatly helps the evaluation of lung and heart states of pneumonia patients. Our sensor device provides a cost-effective alternative approach to the diagnosis and prognosis of pneumonia diseases or other similar diseases and has great potential for clinical use and home-use health monitoring.

\section{Materials and methods}

\section{Sensor design and working principles}

We designed a two-stage amplified PZT sensor with high sensitivity for cardiorespiratory sound monitoring (Fig. 1a, b). First, the sound sensor was based on piezoelectric materials and an asymmetric gapped cantilever structure (Fig. 1c, d), which was composed of a bottom mechanical layer and a top piezoelectric layer separated by a gap (Fig. 1d and Table 1 ). The top piezoelectric layer was made from ceramic PZT (lead zirconium titanate, $\mathrm{Pb}$ $\left.\left(\mathrm{Zr}_{x} \mathrm{Ti}_{(1-x)}\right) \mathrm{O}_{3}\right)$, a widely used piezoelectric material ${ }^{28}$. Due to the piezoelectric effect of PZT materials, the strain on the piezoelectric beam could be transferred into electric charge $^{29}$. Second, a built-in charge amplifier circuit was designed to further amplify the electric signal produced by the piezoelectric beam (Fig. 1e). A $1 \mathrm{G} \Omega$ resistor $\left(R_{F}\right)$ and a $47 \mathrm{pF}$ capacitor $\left(C_{F}\right)$ were used as the feedback resistor and feedback capacitor, respectively. An ultralow input bias current operational amplifier (LMP7721, Texas Instruments) was used to achieve the best performance of the sensor (Fig. 1e). To minimize the surface current leakage, a guard trace was designed to completely surround the input terminal and other circuitry connecting to the inputs of the operational amplifier. Together, the fabricated prototype sensor had a total weight of $13.5 \mathrm{~g}$ with a size of $39 \times 23 \times 13.5 \mathrm{~mm}(l \times w \times h)$.

\section{Theoretical simulation of sensor performance}

We used theoretical simulation to estimate sensor performance with different structures: one was the accelerometer with asymmetric gapped cantilever structure in the present study (Table 1), and the other was the conventional cantilever structure (Fig. S1). Harmonic analysis of the dynamic model of different structures was performed in COMSOL Multiphysics ${ }^{\circledR}$ (COMSOL Inc.), with the parameters set as described in Table 1. The amplitude-frequency of the accelerometers under different excitation forces were simulated. The amplitude was then converted to the strain, and the strain on the piezoelectric beam was plotted over the frequency with variable excitation forces.

\section{Measurement of sensitivity of our sensor}

We characterized the frequency response of our sensor using a mechanical shaker (ET-126B, Labworks). We used a commercial accelerometer (752A13, Endevco) as the gold standard sensor. The outputs of both sensors were voltage, which was recorded by a 16-bit data acquisition board (NI USB 6210, National Instrument) simultaneously. The voltages were recorded by setting the shaker to different frequencies (0 to $2000 \mathrm{~Hz})$ and different accelerations. The sensitivity of the sensors was calculated over different frequencies.

\section{Collection of lung and heart signal data}

We recorded the lung and heart sounds of healthy volunteers $(n=5)$ and discharged pneumonia patients during the follow-up visit in the hospital $(n=41)$. The pneumonia patients who met the discharge criteria were discharged from the hospital. At different times after discharge (in weeks), the patients visited the hospital for a follow-up examination, and we monitored the lung and heart sounds of patients during their follow-up visit.

During sound monitoring, we chose the device location to the right anterior intercostal space above the level of the third rib for respiratory signal detection and the fifth intercostal space to the left immediately lateral to the sternum for cardiac signal detection (Fig. 1a). We recorded the lung and heart sounds for $60 \mathrm{~s}$ for each assay. In this study, data were recorded from 41 pneumonia patients who were recently discharged between 15 June and 2 September 2020. If the same patient was monitored several times on different dates, only the data from the first monitoring session were used for analysis of type classification.

For comparison, we also monitored the lung and heart sounds using a commercial high-end electronic stethoscope (3 M Littman 3200), and the results of both devices were compared.

\section{Data processing}

We transferred the collected data from the sound sensors to a computer through a data acquisition board (NI USB 
6210), and we further processed the data by LabVIEW ${ }^{\circledR}$ and $M_{A T L A B}{ }^{\circledR}$. We fixed the sampling rates to be $6 \mathrm{kHz}$. For data treatment, we applied a filter with a bandwidth from 20 to $400 \mathrm{~Hz}$ to extract heart sounds and applied a filter with a bandwidth from 60 to $1000 \mathrm{~Hz}$ to extract lung sounds.

\section{Statistical analysis}

No statistical methods were used to predetermine sample sizes. The experiments were not randomized, and investigators were not blinded during experiments and outcome assessment. Data are presented as the mean \pm standard deviation (SD). Statistical analysis was performed using Student's $t$-test, and a $p$ value less than 0.05 was considered statistically significant.

\section{Acknowledgements}

This study was supported by the National Natural Science Foundation of China (51727901, 81871484). We thank Dr. Yong Xu of Wayne State University for his guidance in the development of the sensor. We thank the workers at Renmin Hospital, Renmin Hospital East campus, and the Ninth Hospital of Wuhan for their support of this work.

\section{Author details}

'Department of Pulmonary and Critical Care Medicine, Renmin Hospital of Wuhan University, Wuhan 430060, China. ${ }^{2}$ School of Mechanical Science and Engineering, Huazhong University of Science and Technology, Wuhan 430074, China. ${ }^{3}$ School of Power and Mechanical Engineering \& the Institute of Technological Science, Wuhan University, Wuhan 430072, China. ${ }^{4}$ Department of Thoracic, Renmin Hospital of Wuhan University, Wuhan 430060, China. ${ }^{5}$ Department of Pulmonary and Critical Care Medicine, the Ninth Hospital of Wuhan, Wuhan 430081, China. 'SChool of Microelectronics, Wuhan University, Wuhan 430072, China

\section{Author contributions}

S.L. proposed the study; S.L. and Y.L. designed the experiments; H.C. managed the patient contacts; S.L. and S.Y. developed the sensor prototype; S.Y. proposed the signal processing algorithm and performed data processing; H.L. helped in the preparation of the figures; J.L. carried out the theoretical simulation; Y.X. and D.W. helped in the management of patients; X.P. and C.Z. collected the patient information and assisted in the follow-up visit of patients; S.Y. and Y.L. collected the raw sensor monitoring data, evaluated the data and interpreted the results; $Y$. L. wrote the initial draft of the manuscript; S.L. revised the manuscript; all authors read and approved the submission of the manuscript.

\section{Conflict of interest}

The authors declare no competing interests.

Supplementary information The online version contains supplementary material available at https://doi.org/10.1038/s41378-021-00274-x.

Received: 23 January 2021 Revised: 15 March 2021 Accepted: 27 April 2021 Published online: 22 July 2021

\section{References}

1. Huang, C. et al. Clinical features of patients infected with 2019 novel coronavirus in Wuhan, China. Lancet 395, 497-506 (2020).
2. Chung, M. et al. CT imaging features of 2019 novel coronavirus (2019-nCoV). Radiology 295, 202-207 (2020).

3. Pan, F. et al. Time course of lung changes at chest $C T$ during recovery from coronavirus disease 2019 (COVID-19). Radiology 295, 715-721 (2020).

4. Groves, A. M. et al. Idiopathic pulmonary fibrosis and diffuse parenchymal lung disease: implications from initial experience with F-18-FDG PET/CT. J. Nucl. Med. 50, 538-545 (2009).

5. Christe, A. et al. Computer-aided diagnosis of pulmonary fibrosis using deep learning and CT images. Investig. Radiol. 54, 627-632 (2019).

6. Kermany, D. S. et al. Identifying medical diagnoses and treatable diseases by image-based deep learning. Cell 172, 1122-1131.e1129 (2018).

7. Bataille, B. et al. Integrated use of bedside lung ultrasound and echocardiography in acute respiratory failure a prospective observational study in ICU. Chest 146, 1586-1593 (2014).

8. Inciardi, R. M. et al. Cardiac involvement in a patient with coronavirus disease 2019 (COVID-19). JAMA Cardiol. 5, 819-824 (2020).

9. Puntmann, V. O. et al. Outcomes of cardiovascular magnetic resonance imaging in patients recently recovered from coronavirus disease 2019 (COVID19). JAMA Cardiol. 5, 1265-1273 (2020).

10. Guo, Z., Liu, H., Dai, W. \& Lei, Y. Responsive principles and applications of smart materials in biosensing. Smart Mater. Med. 1, 54-65 (2020).

11. Wang, X. W., Gu, Y., Xiong, Z. P., Cui, Z. \& Zhang, T. Silk-molded flexible, ultrasensitive, and highly stable electronic skin for monitoring human physiological signals. Adv. Mater. 26, 1336-1342 (2014).

12. Troiano, R. P. et al. Physical activity in the United States measured by accelerometer. Med. Sci. Sports Exerc. 40, 181-188 (2008).

13. Yang, C. C. \& Hsu, Y. L. A review of accelerometry-based wearable motion detectors for physical activity monitoring. Sensors 10, 7772-7788 (2010).

14. $\mathrm{Hu}, \mathrm{Y}$. \& Xu, Y. An ultra-sensitive wearable accelerometer for continuous heart and lung sound monitoring. In Proc. 2012 Annual International Conference of the IEEE Engineering in Medicine and Biology Society, 694-697 (2012).

15. $\mathrm{Fu}, \mathrm{X}$. et al. A novel sound sensor and its package used in lung sound diagnosis. In Proc. 2014 IEEE 64th Electronic Components and Technology Conference (ECTC), 1189-1191 (2014).

16. Hu, Y. T., Kim, E. G., Cao, G., Liu, S. \& Xu, Y. Physiological acoustic sensing based on accelerometers: a survey for mobile healthcare. Ann. Biomed. Eng. 42 , 2264-2277 (2014).

17. Han, X. et al. Novel resonant pressure sensor based on piezoresistive detection and symmetrical in-plane mode vibration. Microsyst. Nanoeng. 6, 95 (2020)

18. Hodjat-Shamami, M. \& Ayazi, F. Eigenmode operation of piezoelectric resonant gyroscopes. Microsyst. Nanoeng. 6, 108 (2020).

19. Li, Y. F., Zheng, Q. L., Hu, Y. T. \& Xu, Y. Micromachined piezoresistive accelerometers based on an asymmetrically gapped cantilever. J. Microelectromech. Syst. 20, 83-94 (2011).

20. Kim, J. H. et al. Fabrication process of PZT piezoelectric cantilever unimorphs using surface micromachining. Integr. Ferroelectr. 15, 325-332 (1997).

21. DeVoe, D. L. \& Pisano, A. P. Surface micromachined piezoelectric accelerometers (PiXLs). J. Microelectromech. Syst. 10, 180-186 (2001).

22. Beliveau, A., Spencer, G. T., Thomas, K. A. \& Roberson, S. L. Evaluation of MEMS capacitive accelerometers. IEEE Des. Test. Comput. 16, 48-56 (1999).

23. Resnekov, L. Understanding heart sounds and murmurs, with an introduction to lung sounds. JAMA 254, 124-125 (1985).

24. Semmlow, J. \& Rahalkar, K. Acoustic detection of coronary artery disease. Annu. Rev. Biomed. Eng. 9, 449-469 (2007).

25. Sonnweber, T. Cardiopulmonary recovery after COVID-19-an observational prospective multi-center trial. Eur. Respir. J. 57, 2003481 (2021).

26. Zhou, H. et al. Stretchable piezoelectric energy harvesters and self-powered sensors for wearable and implantable devices. Biosens. Bioelectron. 168, 112569 (2020).

27. Kim, J., Campbell, A. S., de Ávila, B. E.-F. \& Wang, J. Wearable biosensors for healthcare monitoring. Nat. Biotechnol. 37, 389-406 (2019).

28. Saito, Y. et al. Lead-free piezoceramics. Nature 432, 84-87 (2004).

29. Wang, Z. L. \& Song, J. Piezoelectric nanogenerators based on zinc oxide nanowire arrays. Science 312, 242-246 (2006). 